\title{
Transitory Inhibition of the Left Anterior Intraparietal Sulcus Impairs Joint Actions: A Continuous Theta-Burst Stimulation Study
}

\author{
Lucia M. Sacheli ${ }^{1,2}$, Gaetano Tieri ${ }^{1,3}$, Salvatore M. Aglioti ${ }^{1,4}$, and Matteo Candidi ${ }^{1,4}$
}

\begin{abstract}
Although temporal coordination is a hallmark of motor interactions, joint action (JA) partners do not simply synchronize; rather, they dynamically adapt to each other to achieve a joint goal. We created a novel paradigm to tease apart the processes underlying synchronization and JA and tested the causal contribution of the left anterior intraparietal sulcus (aIPS) in these behaviors. Participants had to synchronize their congruent or incongruent movements with a virtual partner in two conditions: (i) being instructed on what specific action to perform, independently from what action the partner performed (synchronization), and (ii) being instructed to adapt online to the
\end{abstract}

\section{INTRODUCTION}

When people interact to achieve a common goal, they need to adjust to their partner's movements. For example, a musical ensemble in which musicians ignore the movements of their conductor will achieve a lower and less pleasant performance (D'Ausilio et al., 2012). Despite their importance in many real-life social circumstances, the mechanisms that allow people to implement interactive reciprocal adaptation are not yet fully understood. Here, we aim to test the hypothesis that the left anterior intraparietal sulcus (aIPS) plays a causal role in supporting smooth interpersonal coordination by allowing the integration of the observed partner's action within one's own motor plan. This integration process is the sensorimotor backbone of joint actions (JAs), meaning situations where two or more agents coordinate their actions to achieve a change in the environment together (Sebanz, Bekkering, \& Knoblich, 2006). During a JA, coagents need to organize their individual subgoals to achieve a goal that cannot be achieved alone (Sacheli, Aglioti, \& Candidi, 2015). By definition, JAs imply that each agent's action depends on the partner's one: This interdependence between coagents is what singles out JAs from situations in which people merely

${ }^{1}$ IRCCS Fondazione Santa Lucia, Rome, Italy, ${ }^{2}$ University of Milano-Bicocca, ${ }^{3}$ University UNITELMA Sapienza, "“'Sapienza” University of Rome partner's action (JA). Offline noninvasive inhibitory brain stimulation (continuous theta-burst stimulation) over the left aIPS selectively modulated interpersonal synchrony in JA by boosting synchrony during congruent interactions and impairing it during incongruent ones, while leaving performance in the synchronization condition unaffected. These results suggest that the left aIPS plays a causal role in supporting online adaptation to a partner's action goal, whereas it is not necessarily engaged in social situations where the goal of the partner is irrelevant. This indicates that, during JAs, the integration of one's own and the partner's action goal is supported by aIPS. act independently but "one next to each other." In this domain, it is thus essential to devise interactive experimental paradigms where the agent's subgoal is inherently linked to, and dependent on, the one of the partner. Although synchronization might be fundamental in many social situations, synchronic JAs allow studying the contribution of specific neural substrates to the online control of actions that are constrained by both the partner's actions and the JA goal.

Importantly, this definition of JA holds in the case of both imitative and complementary actions, namely, when partners are required to perform either congruent or incongruent movements, for example, when shaking hands (imitative JA) or passing/receiving an object (complementary JA). Both situations require fine-tuned coordination in time and space, which would never be accomplished by merely reacting to the partner's movements. Accordingly, any JA entails predictive motor processes (Vesper, van der Wel, Knoblich, \& Sebanz, 2013; Knoblich \& Jordan, 2003): Agents base their own behavior on predictions about the partner's behavior, thus this becomes an integral part of the agent's motor plan. Both imitative and complementary JAs rely on the recruitment of a frontoparietal network (Newman-Norlund, van Schie, van Zuijlen, \& Bekkering, 2007) and show similar performance levels in experimental contexts (Ocampo \& Kritikos, 2010; van Schie, van Waterschoot, \& Bekkering, 2008). Crucially, however, in certain circumstances, 
automatic simulation of the partners' actions might suffice to support imitative JAs (Heyes, 2011; Iacoboni, 2005). In contrast, simulation of others' actions becomes detrimental during the execution of complementary JAs, where the coupling of two different motor representations (i.e., the observed one and the executed one) needs to be managed (see Sacheli, Tidoni, Pavone, Aglioti, \& Candidi, 2013). Complementary JAs thus represent the ideal experimental test case to study the underlying mechanisms of partners' action integration and their neural substrates (see also Kokal, Gazzola, \& Keysers, 2009).

We recently showed that the left aIPS plays an important role in supporting complementary JAs (Sacheli, Candidi, Era, \& Aglioti, 2015). On the one hand, aIPS is a visuomotor area responsible for coding action goals during both action execution (Tunik, Frey, \& Grafton, 2005) and action observation (Hamilton \& Grafton, 2006), and it is therefore a good candidate to integrate motor representations that couple one's own goal and a partner's action goal. On the other hand, aIPS is also responsible for filtering visual information and biasing visual attention (Wang et al., 2010; Corbetta \& Shulman, 2002): It might thus well be that its involvement in complementary JAs reflects more general attentional processes, linked to the need to bias visual perception to select task-relevant information while ignoring perceptually salient but irrelevant ones, like the spatial features of the partner's action. The latter explanation would support the idea that complementary JAs are just like any other conflicting context that requires managing different sources of information to perform goal-directed actions (see, for instance, Cross \& Iacoboni, 2014). Thus, if this was the case, one should expect that synchronizing to others' behavior while performing actions that are incongruent with the ones observed in the partner, both when the interaction requires to adapt online to the partner's subgoal (e.g., in complementary JA) and when it does not, would engage aIPS to the same extent and would be equally impaired by the interference with its activity. Conversely, if aIPS interference only impacts complementary JAs, this would suggest that this region is not involved in managing incongruence per se: Rather, this result would suggest that aIPS is essential in integrating the goal of the partner's action within the agent's motor planning process. A clarification of the role of aIPS in complementary JAs would thus critically contribute to identify the cognitive processes at the bases of JAs themselves: It would indicate whether they should be conceptualized as specific cases of conflict monitoring or as goal-based motor processes that allow integrating predictions about a partner's action goal within the agent's motor plan.

On the basis of this background, we aimed to investigate for the first time whether the causal involvement of aIPS is unique to JA and does not generalize to other conflicting social situations in which, for instance, people independently but synchronously act one in front of the other. We thus conceived two experimental conditions, which were identical in terms of motor constrains and visual information conveyed but which differed in the nature of the link between the individual's action goal and the partner's action goal (see Figure 1): Whereas in the Synchronization (Synchr) condition, participants needed to synchronize with the virtual partner independently from the partner's goal, in the JA condition, they needed to synchronize and adapt online to the partner's action goal. Importantly, both conditions entailed congruent and incongruent actions, that is, situations characterized by the absence (congruent) or presence (incongruent) of conflicting visuomotor information. By doing so, we could not only tease apart the role of the left aIPS in JA and Synchr but also clarify the key mechanisms possibly
Figure 1. The figure illustrates the rationale behind the differentiation between the Joint Action (JA) and Synchronization (Synchr) conditions. In the Synchr condition, participants are instructed to synchronize with the partner while achieving an individual goal (e.g., "grasp the lower/upper part of the object"): They may thus in principle ignore what action the partner is doing and focus only on the temporal aspect of his or her movement. On the contrary, in the JA condition, participants need to represent what the partner is doing (i.e., his or her subgoal) and take it into account as a part of their overarching JA goal (i.e., be complementary or imitative as a couple).

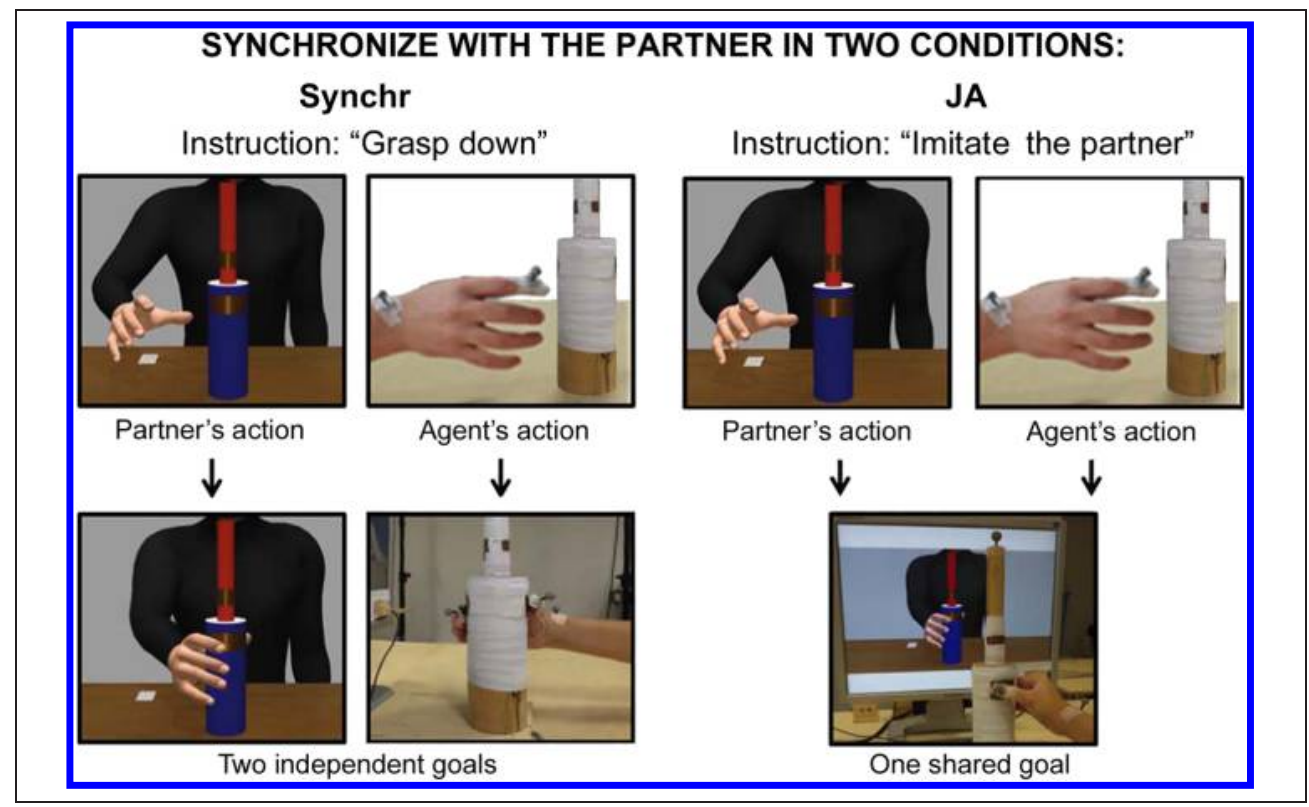


underlying JA itself, that is, conflict monitoring versus self-other action integration. Synchrony with the partner (i.e., grasping asynchrony [GAsynchr]) was measured as an index of interaction performance, whereas movement kinematics was monitored to measure the possible impact of aIPS inhibition on movement execution and to rule out that it might account for the results emerging from the analysis of interaction performance. On the basis of our previous study (Sacheli, Candidi, et al., 2015), we expected interaction performance, indexed by synchrony with the partner, to be selectively modulated by aIPS inhibition: A specific effect of aIPS inhibition in JA would indicate that the role of aIPS does not generalize to other social situations in which conflicting visuomotor processes are at play.

\section{METHODS}

\section{Participants}

Nineteen individuals took part in the study (six men, age $=23.6 \pm 3.2$ years). All participants were righthanded as confirmed by the Standard Handedness Inventory (Briggs \& Nebes, 1975), reported normal or corrected-to-normal vision, and were naive as to the purpose of the experiment. The experimental protocol was approved by the ethics committee of the Fondazione Santa Lucia and was carried out in accordance with the ethical standards of the 1964 Declaration of Helsinki and later amendments. None of the participants had neurological or psychiatric disorder, other medical problems, or any contraindication to TMS (Rossi et al., 2009). Participants gave their written informed consent to take part in the study, received a reimbursement for their participation (which partially depended on their performance during the task; see below), and were debriefed as to the purpose of the study at the end of the experimental procedures. No discomfort or adverse effects to repetitive TMS were reported or observed in any of the participants.

\section{Materials}

\section{Experimental Stimuli and Setup}

The experimental setup was similar to the one applied in previous studies from our group (Sacheli, Candidi, et al., 2015; Sacheli, Christensen, et al., 2015). Participants were comfortably seated in front of a rectangular table $(120 \times$ $100 \mathrm{~cm})$ and watched a $1024 \times 768$ resolution LCD monitor placed on the table at a distance of $\sim 60 \mathrm{~cm}$ from their eyes. Participants had to reach and grasp a bottle-shaped object (30-cm total height) composed of two superimposed cylinders (the body and neck of the bottle) with diameters of 7.0 and $2.5 \mathrm{~cm}$, respectively, placed $45 \mathrm{~cm}$ away from the participants and $5 \mathrm{~cm}$ to the right of the midline. To record participants' touch time on the bottle, two pairs of touch-sensitive copper plates (one per each cylinder) were placed at 15 and $23 \mathrm{~cm}$ of the total height of the object. Before each trial, participants positioned their right hand on a start button placed at a distance of $40 \mathrm{~cm}$ from the bottle-shaped object and $10 \mathrm{~cm}$ to the right of the midline, with their index finger and thumb gently opposed. Given the dimensions of the bottle-shaped object, grasping the lower or upper part would imply a whole-hand grasping (power grip) or a thumb-index finger grasping (precision grip), respectively. Importantly, power and precision grips can be considered different actions as they are dissociable under both the neurophysiological and cognitive points of view (Grafton, 2010; Castiello, 2005).

Auditory instructions were delivered to participants via headphones. They consisted of four sounds having the same intensity ( $4 \mathrm{~dB})$ and duration (100 msec): (i) "high pitched," $1479 \mathrm{~Hz}$; (ii) "low pitched," $115.5 \mathrm{~Hz}$; (iii) "op" (abbreviation of "opposite," "opposto" in Italian); and (iv) "ug" (abbreviation of "same," "uguale" in Italian). The feedback signals about participants' performance were provided via a green/red LED light placed next to the bottom right corner of the screen.

\section{Creation of Virtual Interaction Partner}

Figure 2A shows a graphical description of how videos representing the virtual partner's movements were created. Videos showed the upper body of a character (see also Tieri, Tidoni, Pavone, \& Aglioti, 2015) performing naturalistic grasping movements toward the upper (precision grip) or lower (power grip) part of the bottle-shaped object (https://agliotilab.org/sacheli-tieri-aglioti-candidi2018). Motion capture data were applied on the virtual character by means of MotionBuilder 2011 (Autodesk, Inc., San Rafael, CA) and then rendered in $3 \mathrm{ds}$ Max 2011. The complete sample of videos was composed of 14 different grasping movements (seven power and seven precision grips). Four additional "correction" movements were created by editing the key frame sequence in $3 \mathrm{~d}$ s Max, where the initial and final parts of the power and precision grips were merged resulting in grasping movements in which the virtual arm shifts from a precision grip to a power grip (or vice versa) at the middle of the total length of the movement. Each clip lasted $\sim 3 \mathrm{sec}$ for each experimental condition.

\section{Procedure}

Participants were required to perform the joint grasping task using their right dominant hand. They had to interact with the virtual partner displayed on the screen during two different conditions (in different experimental blocks):

1. During the Synchr condition, participants received an auditory instruction indicating which part of the object they had to grasp (low-pitched sound = "grasp the lower part" with a power grip; high-pitched 


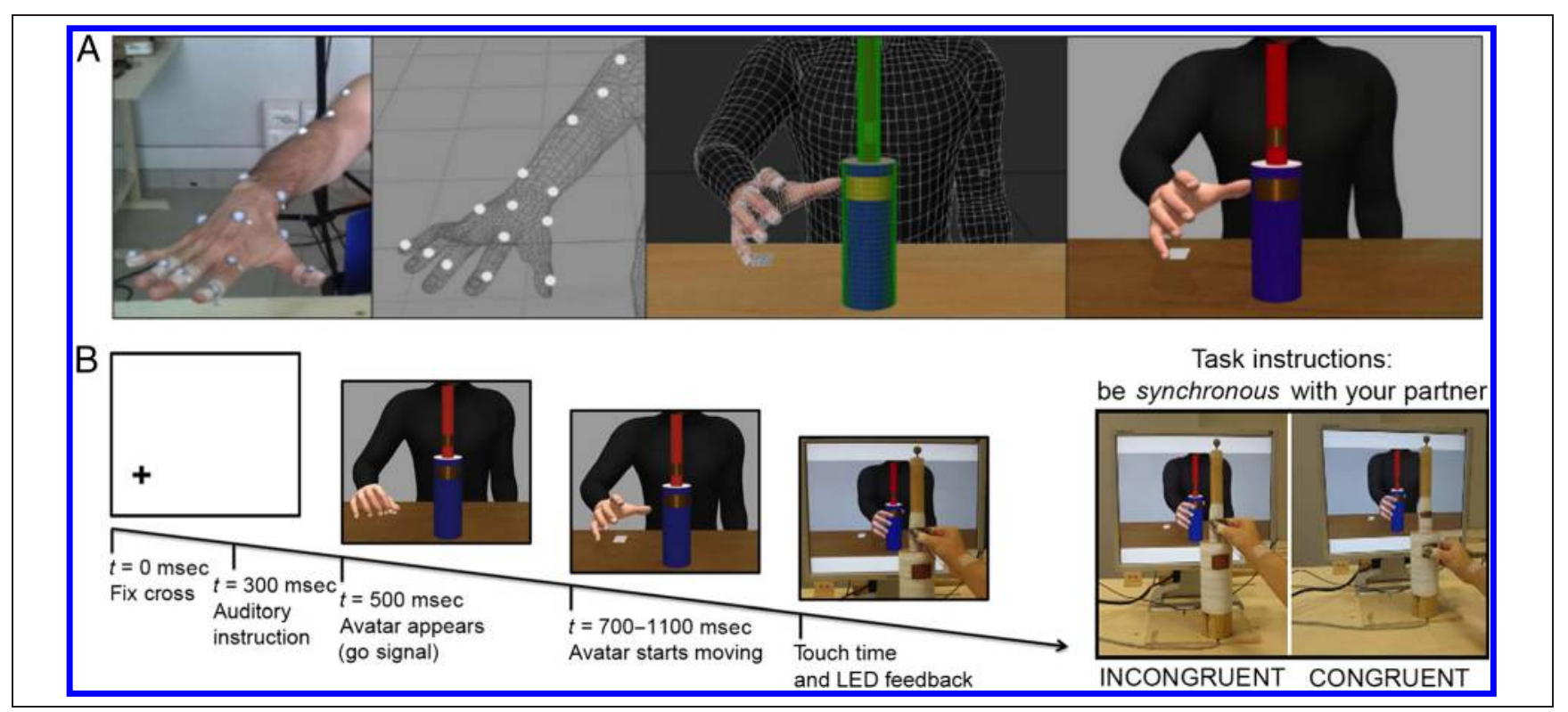

Figure 2. The figure illustrates (A) the infrared motion capture data applied on the virtual partner and (B) the trial timeline. (A) The kinematic features of the virtual interaction partner were based on the movements of a human model performing different grasping movements with the right dominant hand and recorded using 3-D motion capture procedures (SMART-D motion capture system; Bioengineering Technology \& Systems [B $|\mathrm{T}| \mathrm{S}]$ ). The 3-D positions of 19 passive reflecting markers, attached to the actor's upper body (see Tieri et al., 2015), were recorded with a spatial error below 0.5 mm and at a temporal resolution of $100 \mathrm{~Hz}$. Raw data were processed offline to reconstruct and label the markers and to interpolate short missing parts of the trajectories. The final processed trajectories were applied on the virtual character by using commercial software (MotionBuilder 2011; Autodesk, Inc.), whereas the virtual scenario and the character were designed in 3DS Max 2011 and Maya 2011 (Autodesk, Inc.), respectively. (B) The presentation of each clip was preceded by a fixation cross (500 msec) placed on the region of the screen where the partner's hand would appear, which alerted participants about the impending trial. Furthermore, $300 \mathrm{msec}$ after fixation cross onset, the auditory instruction (100 msec) was delivered, and then the partner appeared: This was also the participants' go signal, indicating that they could release the start button. To enhance variability, the partner started his or her movement after a variable delay between 200 and $600 \mathrm{msec}$, mimicking the RTs of human participants recorded during our previous studies applying the same joint-grasping task (Sacheli et al., 2013; Sacheli, Candidi, Pavone, Tidoni, \& Aglioti, 2012).

sound = "grasp the upper part" with a precision grip). Participants were required to grasp the bottle as synchronous as possible with the virtual partner while following these instructions. Thus, they had to synchronize their movements with the partner's movements, yet they could, in principle, ignore where the partner would grasp the bottle. Unbeknownst to participants, instructions led them to perform movements that were $50 \%$ of times congruent and 50\% of times incongruent with the partner's movements, thus replicating the kind of visuomotor incongruence/ congruence present in the JA condition (see below).

2. During the JA condition, an auditory instruction specified whether participants had to perform a complementary ("op" sound, abbreviation for "opposto," i.e., "opposite" in Italian) or imitative ("ug" sound, abbreviation for "uguale," i.e., "same" in Italian) action with respect to the virtual partner. For instance, in imitative trials, if the partner grasped the upper part of the object, participants would also grasp the upper part (congruent movement); on the contrary, in complementary trials, participants would grasp the lower part (incongruent movement; Figure 2B). No information was provided beforehand on which part of the bottle-shaped object they were supposed to grasp (i.e., the upper part with a precision grip or the lower part with a power grip), so that they had to adapt online to the partner.

Synchr and JA were divided in different blocks. Both JA and Synchr blocks included congruent and incongruent trials, in randomized order within the block.

Details on the trial timeline are illustrated in Figure 2B. At the end of each trial, participants received feedback about their performance (the green or red LED light turned on). A "win" trial (green light) required not only that participants correctly followed the auditory instructions, that is, that they correctly grasped the upper/lower part of the bottle (Synchr condition), or performed complementary/imitative movements with respect to the partner (JA condition) but also that they achieved synchrony with the partner. The action was considered synchronous when the time delay between the participant and partner's index-thumb contact times on their bottle fell within a given time window, which was narrowed or enlarged on a trial-by-trial basis according to a staircase procedure. Thus, this procedure allowed tailoring the time window to set GAsynchr on the specific skill of each participant. To motivate individual commitment during the task, participants knew that their monetary reward would depend on the number of wins accumulated during the experimental sessions. Note that the partner's 
index-thumb contact times were measured trial-by-trial by a photodiode placed on the screen that sent a signal recorded by E-Prime2 software (Psychology Software Tools Inc., Pittsburgh, PA). The photodiode was triggered by a black square displayed on the screen (not visible to the participants) at the clip frame corresponding to the instant when the partner grasped his or her virtual object.

Before any brain stimulation and recording of the motor task, a familiarization block of eight trials was delivered.

In each session (i.e., after either continuous thetaburst stimulation [CTBS] of aIPS or sham stimulation), participants performed four Synchr/JA blocks. Block order was counterbalanced between participants and kept constant in the two stimulation sessions in each participant.

Each Synchr/JA block was composed of 32 trials: All power $(n=7)$ and precision $(n=7)$ grip videos were presented twice within the block (i.e., 14 power +14 precision $=28$ trials), $50 \%$ of times in trials requiring a congruent/incongruent response, in randomized order within the block; in addition, four correction trials were included in each block (i.e., $28+4=32$ trials per block). Randomization was controlled by E-Prime2 software so that the same video was never consecutively presented and that corrections could never consecutively occur more than twice. Correction trials were added to ensure that participants adapted online to the partner in the JA condition but were discarded from the analyses; however, mean accuracy was $96 \pm 3 \%$, demonstrating good online coordination. Crucially, in congruent/incongruent and JA/Synchr trials, visual stimuli were identical, and participants watched and synchronized with the same partner's movements.

\section{Movement Kinematics Analysis}

During the whole experiment, the participants' movement kinematics was recorded with infrared cameras to monitor participants' motor execution during the task. Three infrared reflective markers (5-mm diameter) were attached on the participants' right upper limb to the following points: (i) thumb, ulnar side of the nail; (ii) index finger, radial side of the nail; and (iii) wrist, dorso-distal aspect of the radial styloid process. Movement kinematics was recorded with a SMART-D motion capture system (Bioengineering Technology \& Systems [B $|\mathrm{T}| \mathrm{S} \mid]$ ). Four infrared cameras with wide-angle lenses (sampling rate $=$ $100 \mathrm{~Hz}$ ), placed about $100 \mathrm{~cm}$ away from each of the four corners of the table, captured the movements of the markers in 3-D space. The standard deviation of the reconstruction error was always lower than $0.5 \mathrm{~mm}$ for the three axes.

We analyzed participants' reaching trajectory as indexed by the maximum peak of wrist height on the vertical plane $(\mathrm{MaxH})$, and we also measured the maximum peak of wrist velocity on the median plane (MaxV) and the instant at which this peak was reached ( $\mathrm{T}_{-} \mathrm{MaxV}$, trial-by-trial normalized on movement time (MT) so that the final measure was expressed in percentage) to describe the velocity profile of the reaching phase. With regard to the grasping component, we measured maximum grip aperture (MaxAp), that is, the maximum peak of index-thumb 3-D Euclidean distance. For MaxH and MaxAp, we calculated not only the individual mean value of each condition (MaxH and MaxAp) but also the standard deviation of the mean of each condition (SD_MaxH and SD_MaxAp) to measure variability in individuals' reaching and grasping behaviors.

The SMART-D software package (B|T|S|) was used to analyze data and provide a 3-D reconstruction of the marker positions as a function of time. The times of participants' start-button hand release and index-thumb contact times on the bottle were used to subdivide the continuous kinematics recording and analyze only the reach-to-grasp phase.

\section{TMS}

TMS was performed using a 70-mm figure-of-eight coil connected to a Magstim Super Rapid Transcranial Magnetic Stimulator (The Magstim Company, Carmarthenshire, UK). We applied $20 \mathrm{sec}$ of cTBS, which has been shown to have an inhibitory effect over the stimulated site starting from 5 min after stimulation and lasting up to 20 min after stimulation (Huang, Edwards, Rounis, Bhatia, \& Rothwell, 2005). The intensity of stimulation was determined for each participant relative to the participant's resting motor threshold (rMT). To establish participants' rMT, we followed the procedure described below. Participants wore a tightly fitting bathing cap on which scalp stimulation points were marked. Motor evoked potentials (MEPs) were recorded from the first dorsal interosseous muscle of the right hand. Surface $\mathrm{Ag}-\mathrm{AgCl}$ electrodes were placed in a belly-tendon montage with the active electrode placed over the motor point and the reference over the interphalangeal joint. EMG recording was performed with a CED Power 1401 (Cambridge Electronic Design Ltd., Cambridge, UK) connected to an Isolated Patient Amplifier System Model D360 (Digitimer Ltd., Hertfordshire, United Kingdom) and interfaced with CED Spike 2 software. The rMT, defined as the lowest intensity able to evoke 5 of 10 MEPs with an amplitude of at least $50 \mu \mathrm{V}$, was determined by holding the stimulation coil over the optimal scalp position. The optimal scalp position for inducing MEPs in the right first dorsal interosseous muscle was found by moving the coil in steps of $1 \mathrm{~cm}$ over the left primary motor cortex until the largest MEPs were found and then marked with a pen on a bathing cap worn by participants. Mean rMT was $55 \pm 8.1 \%$ of the stimulator output.

The procedure of cTBS was similar to Huang and colleagues (2005) and identical to the one applied by Sacheli, Candidi, and colleagues (2015): Trains of three 
pulses at $50 \mathrm{~Hz}$ were delivered every $200 \mathrm{msec}(5 \mathrm{~Hz})$ for $20 \mathrm{sec}$ (300 pulses in total). cTBS was applied at $80 \%$ of the rMT (mean $=44.1 \pm 6.5 \%$ of the stimulator output). After resting for $5 \mathrm{~min}$ with their right arm relaxed on the side, participants started the joint grasping task. The task never lasted more than 10 min so that the inhibitory time window was never exceeded. We used the SofTaxic Navigator system to identify and store the sites that, according to the coordinates reported by Hamilton and Grafton (2006), were optimally targeting left aIPS (Montreal Neurological Institute coordinates $=-52-32$ 44, converted in Talairach coordinates $=-47-3437$, according to Tunik, Rice, Hamilton, and Grafton [2007]). Skull landmarks (nasion, inion, and two preauricular points) and 61 points providing a uniform representation of the scalp were digitized by means of a Polaris Vicra Optical Tracking System (Northern Digital Inc., Waterloo, Canada). Coordinates in Talairach space (Talairach \& Tournoux, 1988) were automatically estimated by the SofTaxic Navigator from an MRI-constructed stereotaxic template using an individualized probabilistic head model computation. This individualized head model preserves the anatomical scalp-brain correlates of a mean MR template, providing an accurate set of estimated MRI data, specific for the participant under examination. The resulting mean stimulation site was as follows: Talairach coordinates $=-47.4 \pm 1.1,-33.8 \pm 0.9,36 \pm 1.1$ (see Figure 3). Thanks to coil calibration, the navigation system allowed the overlap of the coil focus with these coordinates and made it possible to monitor online any movement of the coil during the $20 \mathrm{sec}$ of cTBS. Displacements from the optimal individual scalp locations for aIPS stimulation never exceeded $2 \mathrm{~mm}$ for any of the three axes. Sham stimulation was used as the control stimulation condition and consisted in cTBS delivered on a 3-cm-thick wooden rectangular-shaped object placed on the vertex of each participant's head. aIPS/sham stimulation was counterbalanced between participants. Between sessions, independently from session order, a break of $1 \mathrm{hr}$ ensured that the effect of stimulation had faded away.

\section{Data Handling and Analyses}

Correct trials only were entered in the behavioral and kinematics analyses. More specifically, we excluded from the analyses trials in which participants (i) missed the touch-sensitive copper plates (and their response was thus not recorded), (ii) started their movements before the go signal (i.e., made false starts), or (iii) did not respect their auditory instructions (on average, excluded trials were $6.64 \pm 4.12 \%$ of the total, equal to $14.88 \pm 9.23$ trials)

We considered the following as measures of performance: (i) accuracy, that is, percentage of correct movements executed according to the instructions (false starts were considered errors), and (ii) GAsynchr, that is, absolute value of time delay between the participant's and partner's index-thumb contact times on the bottle; note that "contact time" is computed for both the participant and the partner as the time from the go signal onset to the instant of their index-thumb contact with their bottle.

As behavioral indexes, we also considered RTs, that is, time from the go signal to the instant participants released the start button, and MTs, that is, time from the instant when participants released the start button to the instant when their fingers touched the bottle. Moreover, we analyzed the kinematic parameters describing the reaching $\left(\mathrm{MaxH}, \mathrm{SD}_{-} \mathrm{MaxH}, \mathrm{MaxV}, \mathrm{T}_{-} \mathrm{MaxV}\right)$ and grasping (MaxAp and SD_MaxAp) components of the reach-to-grasp movement.

For each of the abovementioned behavioral and kinematic measures, we calculated the individual mean in each condition. Values that fell 2.5 SDs

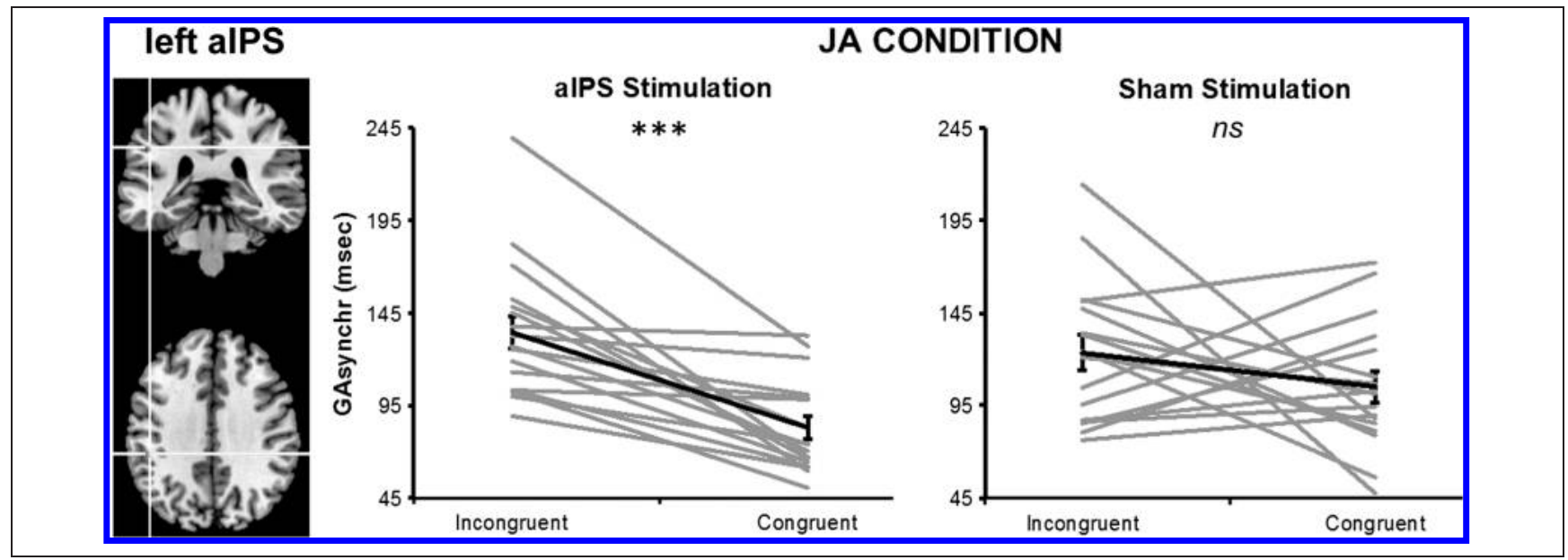

Figure 3. The figure shows (on the left side) the target of aIPS stimulation (mean stimulation site: Talairach $=-47.4 \pm 1.1,-33.8 \pm 0.9,36 \pm 1.1$ ) and (on the right side) the results that emerged from the analysis of participants' interpersonal performance in the JA condition as indexed by GAsynchr (precision grip data only). These results highlight that performance becomes lower (i.e., GAsynchr is higher) in incongruent than congruent actions only after aIPS inhibition, whereas the two conditions do not differ at sham. The gray lines indicate individual data, and the black thick lines indicate mean $( \pm S E M)$ values. The asterisks indicate significant differences: $* * * p<.001$ 
above or below each individual mean for each experimental condition were considered outlier values (on average, outlier trials per variable were $0.64 \pm 0.25 \%$ of the total, corresponding to $1.43 \pm 0.56$ trials). At the group level, participants with an individual mean of 2.5 SDs above or below the group mean were excluded from the analyses: Two participants were outliers on GAsynchr and accuracy according to this criterion and were thus excluded from the analyses (final sample $=17$ participants).

Accuracy data were at ceiling (mean accuracy = $98 \pm 1 \%$ ), and a general linear model was not applicable. We applied a series of nonparametric tests for dependent samples (Wilcoxon signed-rank test) to compare accuracy after aIPS versus sham stimulation in each condition.

The other dependent variables were entered into separate repeated-measures ANOVAs having Stimulation $($ aIPS/Sham $) \times$ Interaction type $(\mathrm{JA} /$ Synchr $) \times$ Congruency (Congruent/Incongruent) $\times$ Movement type (Power/Precision grip) as within-participant factors $(2 \times 2 \times 2 \times 2$ within-participant design).

All tests of significance were based on an $\alpha$ level of .05. Bonferroni correction for multiple comparisons was applied when needed.

As outlined in the Introduction, we expected aIPS inhibition to selectively modulate interaction performance in the JA condition, as measured by GAsynchr. Specifically, we expected aIPS inhibition to make performance in incongruent JA less synchronous than in congruent JA, whereas we expected that this effect would not generalize to the Synchr condition. To show that a possible lack of significant effect of stimulation in Synchr provides evidence in favor of a null effect of aIPS inhibition in this condition, Bayesian statistics were applied, as implemented in JASP (Love et al., 2015). The rationale of this analysis is to consider the Bayes Factor $\left(\mathrm{BF}_{10}\right)$ as a statistical metric that quantifies the strength of evidence that the data provide in favor of the alternative hypothesis relative to the null hypothesis. $\mathrm{BF}_{10}$ higher than 3 indicates strong support for the alternative hypothesis, whereas $\mathrm{BF}_{10}$ lower than 0.3 indicates strong support for the null hypothesis (Rouder, Morey, Verhagen, Swagman, \& Wagenmakers, 2017).

\section{RESULTS}

\section{Interaction Performance: Accuracy and GAsynchr}

\section{Accuracy}

Accuracy data are reported in Table 1. Nonparametric tests indicated that accuracy did not differ after aIPS versus sham stimulation in any condition (all $p \mathrm{~s}_{\text {uncorr }}>.09$ ).

\section{GAsynchr}

The ANOVA on GAsynchr showed a significant main effect of Interaction type $\left(F(1,16)=8.56, p=.010, \eta_{\mathrm{p}}^{2}=\right.$ $.35)$, a significant Interaction type $\times$ Congruency interaction $\left(F(1,16)=7.31, p=.016, \eta_{\mathrm{p}}^{2}=.31\right)$, and a significant Congruency $\times$ Movement type interaction $(F(1$, $\left.16)=31.08, p<.001, \eta_{p}^{2}=.66\right)$ : These effects were further specified by a significant triple Interaction type $\times$ Congruency $\times$ Movement type interaction $(F(1,16)=$ $\left.11.63, p=.004, \eta_{\mathrm{p}}^{2}=.42\right)$, indicating that the strong Congruency $\times$ Movement type interaction effect was modulated by Interaction type. Finally, the ANOVA also showed a highly significant Stimulation $\times$ Interaction type $\times$ Congruency $\times$ Movement type interaction $(F(1$, 16) $\left.=17.07, p<.001, \eta_{\mathrm{p}}^{2}=.52\right)$.

To make this higher-level interaction easier to interpret, we applied a hierarchical approach and performed two follow-up ANOVAs, separately for each Interaction type (JA and Synchr).

The ANOVA on the JA task showed a significant main effect of Congruency $\left(F(1,16)=6.40, p=.022, \eta_{\mathrm{p}}^{2}=\right.$ .29) and a significant Congruency $\times$ Movement type interaction $\left(F(1,16)=7.51, p=.014, \eta_{\mathrm{p}}^{2}=.32\right)$, which were further explained by a significant Stimulation $\times$ Congruency $\times$ Movement type interaction $(F(1,16)=$ $\left.18.68, p<.001, \eta_{\mathrm{p}}^{2}=.54\right)$. The triple interaction indicates that Stimulation modulated the effect of Congruency differently in precision and power grips. Whereas the follow-up ANOVA performed on JA power grip data showed no significant effects (all $p s>1$ ), suggesting no difference in GAsynchr between Congruent and Incongruent actions in this condition, the follow-up ANOVA performed on JA precision grip data showed a significant main effect of Congruency $(F(1,16)=10.81$, $\left.p=.005, \eta_{\mathrm{p}}^{2}=.40\right)$ further specified by the significant

Table 1. Participants' Accuracy in All Experimental Conditions

\begin{tabular}{|c|c|c|c|c|c|c|c|c|c|}
\hline & & $\begin{array}{l}\text { JA-Incongr- } \\
\text { Power }\end{array}$ & $\begin{array}{c}\text { JA-Incongr- } \\
\text { Precision }\end{array}$ & $\begin{array}{l}\text { JA-Congr- } \\
\text { Power }\end{array}$ & $\begin{array}{l}\text { JA-Congr- } \\
\text { Precision }\end{array}$ & $\begin{array}{l}\text { Synchr- } \\
\text { Incongr- } \\
\text { Power }\end{array}$ & $\begin{array}{l}\text { Synchr- } \\
\text { Incongr- } \\
\text { Precision }\end{array}$ & $\begin{array}{l}\text { Synchr- } \\
\text { Congr- } \\
\text { Power }\end{array}$ & $\begin{array}{c}\text { Synchr- } \\
\text { Congr- } \\
\text { Precision }\end{array}$ \\
\hline \multirow[t]{2}{*}{ aIPS stim } & Median & 1.00 & 1.00 & 1.00 & 1.00 & 1.00 & 1.00 & 1.00 & 1.00 \\
\hline & Range & $0.93-1.00$ & $0.92-1.00$ & $0.92-1.00$ & $0.86-1.00$ & $0.86-1.00$ & $0.83-1.00$ & $0.92-1.00$ & $0.91-1.00$ \\
\hline \multirow[t]{2}{*}{ Sham stim } & Median & 1.00 & 1.00 & 1.00 & 1.00 & 1.00 & 1.00 & 1.00 & 1.00 \\
\hline & Range & $0.86-1.00$ & $0.80-1.00$ & $0.85-1.00$ & $0.85-1.00$ & $0.83-1.00$ & $0.79-1.00$ & $0.93-1.00$ & $0.93-1.00$ \\
\hline
\end{tabular}

No comparison between each aIPS and sham condition is significant. Congr $=$ congruent; Incongr $=$ incongruent; stim $=$ stimulation. 
Stimulation $\times$ Congruency interaction $(F(1,16)=$ 8.25, $\left.p=.011, \eta_{\mathrm{p}}^{2}=.34\right)$. The latter result indicates that, in precision grips, the performance in Congruent and Incongruent actions did not differ after sham stimulation $(p>.4)$, whereas it dissociated after aIPS inhibition $(p<.001)$; in other words, inhibition of aIPS made synchrony in Incongruent actions worse than in Congruent ones, because GAsynchr was higher in Incongruent versus Congruent actions only after aIPS inhibition $(p<.001$; see Figure 3).

On the contrary, the ANOVA on the Synchr condition only showed a significant Congruency $\times$ Movement type interaction $\left(F(1,16)=48.32, p<.001, \eta_{\mathrm{p}}^{2}=.75\right)$, indicating that, in the Synchr condition, performance was higher (i.e., GAsynchr was lower) in the Incongruent as compared with the Congruent condition in power grips $(p<.002)$ and in the Congruent as compared with the Incongruent condition in precision grips $(p<.001)$. Most importantly, this pattern was not modulated by aIPS inhibition, as the ANOVA on GAsynchr in the Synchr condition did not show any significant main effect or interaction with the factor Stimulation (all $p s>.5$; see Figure 4).

Bayesian statistics were then applied to investigate whether the pattern of data provides evidence in favor of a null effect of Stimulation in the Synchr, but not in the JA, condition. First, we performed a Bayesian ANOVA on GAsynchr data in the Synchr condition. As we were specifically interested in the effect of Stimulation independently from the other effects, we set the interaction Congruency $\times$ Movement type as a nuisance effect. Results showed strong evidence in favor of the null hypothesis (all $\left.\mathrm{BF}_{10}<0.07\right)$ for all models including the Stimulation $\times$ Congruency interaction. As a matter of fact, Bayesian paired $t$ tests performed on the effect of interest (i.e., the individual Incongruent - Congruent difference) on data measured in the Synchr condition after aIPS versus sham stimulation also showed evidence in favor of the null hypothesis (all $\mathrm{BF}_{10}<0.3$ ) both when performed separately for the precision grip $\left(\mathrm{BF}_{10}=\right.$ $0.26)$ and power grip $\left(\mathrm{BF}_{10}=0.28\right)$ conditions and when performed on their mean $\left(\mathrm{BF}_{10}=0.26\right)$. On the contrary, the Bayesian analyses performed on GAsynchr in the JA condition (precision grip trials) showed very strong evidence in favor of the alternative hypothesis for the model including the Stimulation $\times$ Congruency interaction $\left(\mathrm{BF}_{10}=417.71\right)$; the Bayesian paired $t$ test performed on the effect of interest (i.e., the individual Incongruent Congruent difference) on data measured in the JA condition after aIPS versus sham stimulation also showed evidence in favor of the alternative hypothesis $\left(\mathrm{BF}_{10}=4.95\right)$.

Thus, Bayesian statistics indicated that the null hypothesis should be favored over the experimental hypothesis with regard to the effects of aIPS stimulation in the Synchr condition and that data provided evidence in favor of the presence of an interaction effect between aIPS inhibition and congruency in the JA condition.

\section{Control Analyses on Individuals' Motor Performance: RTs, MTs, and Kinematics Variables}

The statistics of the results that emerged from the ANOVAs on RTs, MTs, and kinematics variables are reported in Table 2 .

\section{RTs and MTS}

The ANOVA on RTs showed a significant main effect of Interaction type indicating that RTs were longer in JA than in Synchr. This is in line with the fact that, in Synchr, participants already knew what part of the bottle they had to grasp even before hearing the go signal, whereas in JA, they had to wait and see what action the partner was going to do to adapt online their own action. The ANOVA also showed a significant Interaction type $\times$ Congruency $\times$ Movement type interaction; however, the only significant comparisons were between JA

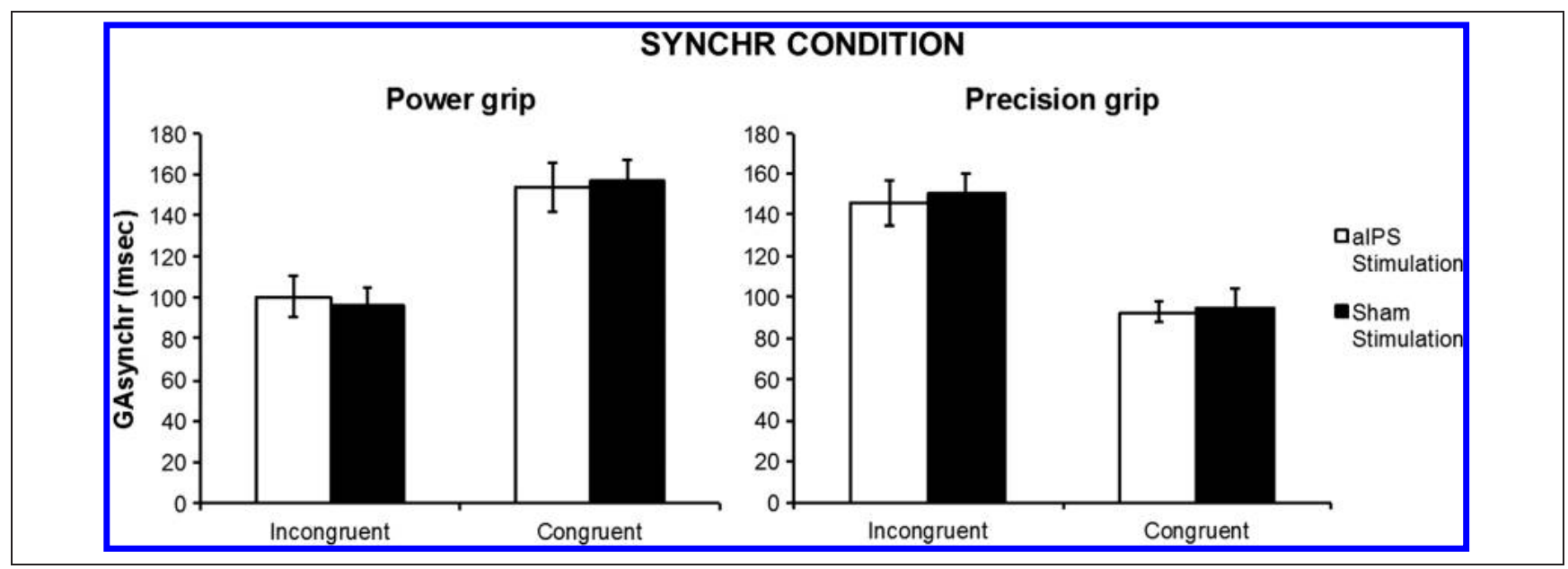

Figure 4. The figure illustrates the pattern of GAsynchr data in the Synchr condition. The results show a significant Congruency $\times$ Movement type interaction, which is completely unaffected by aIPS inhibition. Error bars indicate SEM. 
Table 2. Significant Results That Emerged From the ANOVAs on RTs, MTs, and Kinematics Parameters

\begin{tabular}{|c|c|c|c|c|}
\hline Effect & $F$ & $d f$ & $p$ & $\eta_{p}^{2}$ \\
\hline \multicolumn{5}{|l|}{ RTs } \\
\hline Main effect of Interaction type & 9.83 & 1,16 & .006 & .38 \\
\hline Interaction type $\times$ Congruency $\times$ Movement type & 4.87 & 1,16 & .042 & .23 \\
\hline \multicolumn{5}{|l|}{ MTs } \\
\hline Interaction type $\times$ Congruency & 7.27 & 1,16 & .016 & .31 \\
\hline Interaction type $\times$ Movement type & 31.34 & 1,16 & $<.001$ & .66 \\
\hline \multicolumn{5}{|l|}{$\operatorname{MaxH}$} \\
\hline Main effect of Interaction type & 12.17 & 1,16 & .003 & .43 \\
\hline Main effect of Congruency & 13.92 & 1,16 & .001 & .46 \\
\hline Main effect of Movement type & 858.22 & 1,16 & $<.001$ & .98 \\
\hline Interaction type $\times$ Congruency & 37.81 & 1,16 & $<.001$ & .70 \\
\hline Interaction type $\times$ Movement type & 53.68 & 1,16 & $<.001$ & .77 \\
\hline Congruency $\times$ Movement type & 21.14 & 1,16 & $<.001$ & .57 \\
\hline \multicolumn{5}{|l|}{ MaxH standard deviation (SD_MaxH) } \\
\hline Main effect of Interaction type & 21.48 & 1,16 & $<.001$ & .57 \\
\hline Main effect of Congruency & 8.17 & 1,16 & .011 & .34 \\
\hline Main effect of Movement type & 50.61 & 1,16 & $<.001$ & .76 \\
\hline Interaction type $\times$ Movement type & 20.20 & 1,16 & $<.001$ & .56 \\
\hline Congruency $\times$ Movement type & 13.63 & 1,16 & .002 & .46 \\
\hline Interaction type $\times$ Congruency $\times$ Movement type & 5.69 & 1,16 & .030 & .26 \\
\hline \multicolumn{5}{|l|}{$\operatorname{MaxAp}$} \\
\hline Main effect of Interaction type & 6.71 & 1,16 & .020 & .29 \\
\hline Main effect of Movement type & 260.60 & 1,16 & $<.001$ & .94 \\
\hline Interaction type $\times$ Movement type & 20.08 & 1,16 & $<.001$ & .56 \\
\hline \multicolumn{5}{|l|}{ MaxAp standard deviation (SD_MaxAp) } \\
\hline Main effect of Interaction type & 13.44 & 1,16 & .002 & .46 \\
\hline Main effect of Movement type & 85.73 & 1,16 & $<.001$ & .84 \\
\hline Interaction type $\times$ Movement type & 11.38 & 1,16 & .004 & .41 \\
\hline \multicolumn{5}{|l|}{$\operatorname{Max} V$} \\
\hline Main effect of Interaction type & 50.41 & 1,16 & $<.001$ & .76 \\
\hline Main effect of Movement type & 8.65 & 1,16 & .009 & .35 \\
\hline Congruency $\times$ Movement type & 39.60 & 1,16 & $<.001$ & .71 \\
\hline \multicolumn{5}{|l|}{$T_{-} \operatorname{Max} V$} \\
\hline Main effect of Interaction type & 27.85 & 1,16 & $<.001$ & .63 \\
\hline Stim $\times$ Interaction type $\times$ Congruency $\times$ Movement type & 4.88 & 1,16 & .042 & .23 \\
\hline
\end{tabular}

Importantly, all variables showed an effect of Interaction type. 
and Synchr in each condition (all $p s<.001$ ), whereas all other comparisons were not significant (all $p s>1$ ).

The ANOVA on MTs showed significant Interaction type $\times$ Congruency and Congruency $\times$ Movement type interactions. The first interaction effect indicated that incongruent movements in Synchr took longer times than in JA $(p<.001$, all other $p$ s $>.1)$; the latter indicated that incongruent movements took longer times than congruent movements in power grips $(p=.012)$, whereas the reverse pattern emerged in precision grips (i.e., incongruent movements were shorter than congruent ones, $p=.003$ ).

The ANOVAs on RTs and MTs showed no significant main effect or interaction with the factor Stimulation (all $p s>.2$ ).

\section{Reaching Kinematics (MaxH and SD_MaxH)}

The ANOVA on MaxH showed significant main effects of Interaction type, Congruency, and Movement type, indicating that, overall, MaxH was higher (i) when grasping the higher target location of the bottle (the condition labeled "precision grip") compared with when grasping the lower target location (the condition labeled "power grip"), (ii) during JA compared with Synch, and (iii) during incongruent movements compared with congruent ones. These main effects were further specified by significant second-level interactions (Interaction type $x$ Congruency, Interaction type $\times$ Movement type, and Congruency $\times$ Movement type) indicating that, when participants were grasping the lower target location (the condition labeled "power grip"), wrist height was modulated by the Interaction type and Congruency factors. Indeed, power grips were higher in JA than in Synchr $(p<.001)$ and in incongruent than in congruent movements $(p<.001)$.
Altogether, this pattern of results suggests that, in JA, when participants were grasping the lower target location (the condition labeled "power grip"), their wrist trajectory was influenced by the observation of a (incongruent) higher wrist trajectory in the partner. The lack of modulation in precision grip movements aiming at the higher part of the bottle is likely due to a ceiling effect and in line with previous studies applying the present experimental setup (see Candidi, Curioni, Donnarumma, Sacheli, \& Pezzulo, 2015; Sacheli et al., 2013).

These results were confirmed by the analysis of the standard deviation of MaxH (SD_MaxH), which measured the variability of participants' reaching behavior. The analysis of SD_MaxH also showed a significant main effect of Movement type, which indicates that movements aimed at the lower target location (the condition labeled "power grip") were generally more variable than those aimed at the higher location (the condition labeled "precision grip"). This was explained by the higher-level Interaction type $\times$ Congruency $\times$ Movement type interaction, showing that variability in wrist height when grasping the lower target location (the condition labeled "power grip") was higher during incongruent than congruent actions in the JA condition only $(p<.001)$ : The higher variability of reaching trajectory in JA is likely due to requirement to adapt online to the partner's movements (see Figure 5, right).

The ANOVAs on MaxH and SD_MaxH showed no significant main effect or interaction with the factor Stimulation (all $p s>.1$ ).

\section{Grasping Kinematics (MaxAp and SD_MaxAp)}

The ANOVA on MaxAp showed a significant main effect of Movement type, indicating that MaxAp was larger in power than precision grips, and of Interaction type,

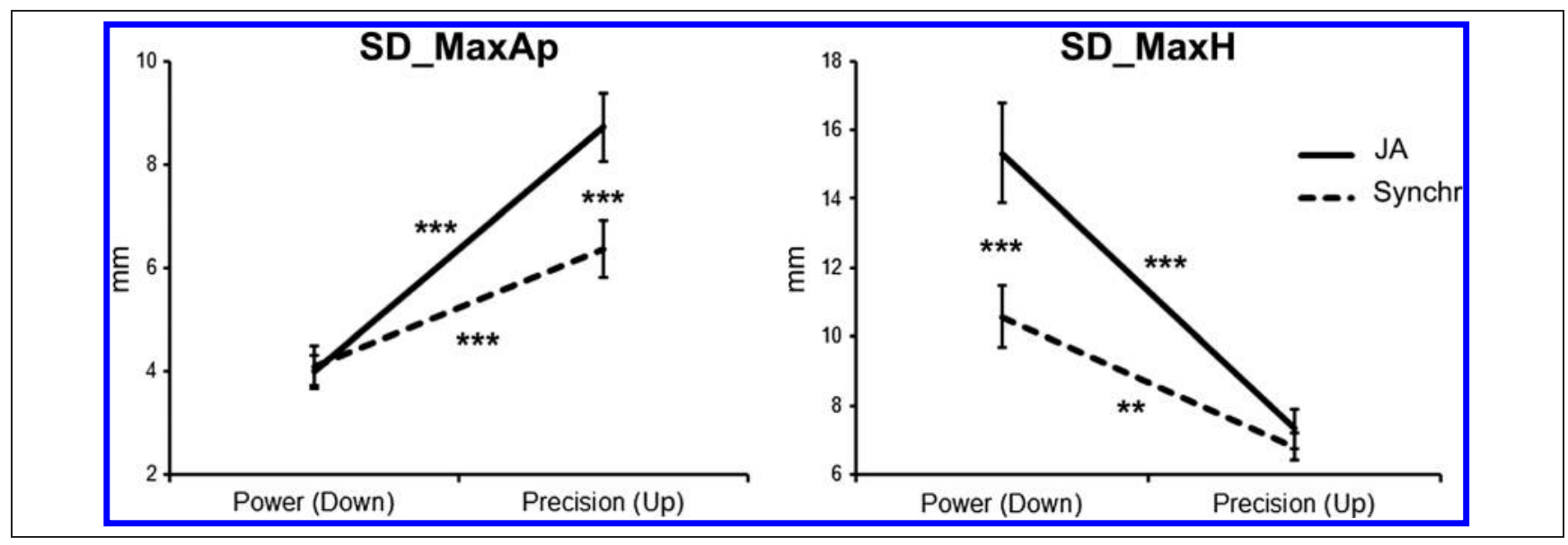

Figure 5. The figure shows the significant Interaction type $\times$ Movement type interaction that emerged from the analysis of variability in participants' reaching and grasping kinematics (i.e., SD_MaxH and SD_MaxAp, respectively). These results demonstrate that the factor Interaction type modulated participants' motor behavior and made it more variable as a consequence of the requirement for online adaptation to the partner's action. Effects are more evident in precision grip (left) for MaxAp and in power grip (right) for MaxH; The lack of modulation in the opposite movements (i.e., in power grip for MaxAp and in precision grip for $\mathrm{MaxH}$ ) is likely due to a ceiling effect and in line with previous studies applying the present experimental setup (see Candidi, Curioni, et al., 2015; Sacheli et al., 2013). ${ }^{* *} p<.01 ; * * *<.001$. 
indicating that, overall, MaxAp in JA was larger than in Synchr. This effect was further explained by a significant Interaction type $\times$ Movement type interaction, indicating that precision grips showed an increase of safety margin in JA as compared with Synchr $(p<$ .001 ), as it might be expected given the higher interdependency between partners' movements required by the JA condition. Power grips did not show such an effect of Interaction type likely because of a ceiling effect of MaxAp in power grips (see also Sacheli, Christensen, et al., 2015).

These results were further confirmed by the analysis of MaxAp standard deviation (SD_MaxAp). Indeed, the ANOVA on SD_MaxAp showed not only a significant main effect of Movement type, indicating that precision grips were generally more variable than power grips, but also a significant effect of Interaction type, indicating that MaxAp in JA was more variable than in Synchr, and a significant Interaction type $\times$ Movement type interaction: It indicates that, although the difference between JA and Synchr was not significant in power grips $(p=.99)$, likely due to a ceiling effect of MaxAp in power grips (see also Sacheli, Christensen, et al., 2015), in precision grips, SD_MaxAp was higher in JA than in Synchr $(p<.001)$, suggesting that participants' MaxAp was more variable in JA due to the need to adapt online to the partner (see Figure 5, left).

The ANOVAs on MaxAp and SD_MaxAp showed no significant main effect or interaction with the factor Stimulation (all $p s>.2$ ).

\section{Velocity Profile (MaxV and T_MaxV)}

The ANOVA on MaxV showed a significant main effect of Interaction type, indicating that peak velocity in the Synchr condition was higher than in the JA condition, as expected given the requirement to adapt online to the partner in JA. The ANOVA also showed a significant main effect of Movement type, indicating that power grips were faster than precision grips, and a Congruency $\times$ Movement type interaction, indicating that peak velocity was higher in congruent than incongruent power grips $(p<.001)$ and in incongruent than congruent precision grips $(p=.006)$ : This effect parallels the one that emerged in the analysis of MTs. The ANOVA on MaxV showed no significant main effect or interaction including the factor Stimulation (all $p s>.1$ ).

The ANOVA on T_MaxV showed a significant main effect of Interaction type, indicating that time to peak velocity in the Synchr condition occurred earlier (at $28.53 \pm 6.32 \%$ of the total MT) than in the JA condition (when it occurred at $32.58 \pm 7.91 \%$ of MT), as expected given the requirement to adapt online to the partner in JA. Moreover, the ANOVA showed a significant Stimulation $\times$ Interaction type $\times$ Congruency $\times$ Movement type interaction. To make this fourth-level interaction easier to interpret, we ran two follow-up ANOVAs sepa- rately for the JA and Synchr conditions. The ANOVA on JA data showed no significant effect (all $p s>.1$ ), whereas the ANOVA on Synchr only showed a significant main effect of Stimulation $(F(1,16)=8.98, p=$ $\left..009, \eta_{\mathrm{p}}^{2}=.36\right)$, indicating that, after aIPS inhibition, participants achieved their reaching peak velocity earlier than after sham stimulation. No other effect was statistically significant. Importantly, although T_MaxV was the only kinematic parameter showing a modulation of participants' behavior induced by aIPS stimulation, it only affected the Synchr condition (by generally anticipating the time of peak, thus possibly reflecting an alerting effect) but not the JA condition. Thus, this result cannot explain the one that emerged from the analysis of interpersonal performance as measured by GAsynchr.

\section{DISCUSSION}

The aim of this study was to investigate the role of aIPS in JAs by comparing the effect of its inhibition in two experimental conditions: Whereas, in the JA condition, participants were required to synchronize by adapting online their subgoal to the partner's (i.e., selecting which part of the bottle-shaped object they had to grasp depending on the action of the partner), in the Synchr condition, participants were required to synchronize but chose their individual subgoal by independently selecting the appropriate grasp type and trajectory. Results showed that left aIPS inhibition modulates interaction performance in the JA condition only, leading interpersonal synchrony in congruent (imitative) and incongruent (complementary) movements to dissociate, although aIPS inhibition had no effects on the nonjoint Synchr condition. Importantly, this result emerged although, at baseline (i.e., after sham stimulation), congruent and incongruent JAs did not show significant differences in performance, in line with previous studies (Sacheli, Candidi, et al., 2015; Ocampo \& Kritikos, 2010; van Schie et al., 2008). This evidence rules out that task complexity per se could account for the selective effect of cTBS found in the JA condition.

The present results suggest that the causal role of aIPS in JAs does not generally reflect the need for visuomotor conflict monitoring (which is equally present in incongruent-Synchr and incongruent-JA condition) and/or attentional processes aimed at filtering visual information; rather, it is specifically linked to the need to integrate the (predicted) goal of the partner's action in one's own motor planning, allowing for online adaptation. Importantly, control analyses performed with Bayesian statistics demonstrate that there is strong evidence (given the data) in favor of a null effect of Stimulation in the Synchr condition, whereas there is strong evidence in favor of the presence of a Stimulation $x$ Congruency interaction effect in JA. 


\section{Synchr vs. JA: Two Different Types of Interaction}

On the basis of our previous study (Sacheli, Candidi, et al., 2015), we expected interaction performance (as indexed by GAsynchr) to be the crucial variable to measure the impact of aIPS inhibition on participants' behavior, whereas we did not expect stimulation to modulate participants' motor performance per se. However, the analysis of participants' RTs, MTs, and kinematics allowed us to test whether, independently from the lack of significant effect of stimulation, JA and Synchr might be considered two different types of interaction. Results support such an assumption, because they showed that participants modulated their behavior depending on which condition they were interacting in, suggesting that different cognitive-motor processes might support JA and Synchr. In fact, in the JA condition, participants showed longer RTs, suggesting that they were waiting for the partner to start their actions; moreover, in JA, participants were more influenced by the partner's behavior, as their wrist kinematics (indexed by $\mathrm{MaxH}$ ) and grip aperture (indexed by MaxAp) showed higher variability and an increase of safety margin: This suggests that participants applied a more careful action planning in JA compared with the Synchr condition and that they were possibly more influenced by the behavior observed in their partner. This was also confirmed by the results that emerged from the analysis of participants' wrist velocity profile, which were compatible with a more feedbackbased motor execution in the JA as compared with the Synchr condition.

Specifically, we suggest that, in JA, the participants might have applied sensorimotor simulative mechanisms (Keysers \& Gazzola, 2014) to predict the goal of the partner's behavior (see Vesper et al., 2013; Bekkering et al., 2009), thus being influenced by the partner's kinematics. By contrast, unwanted simulation of the partner behavior in the Synchr condition would be regulated by top-down control (e.g., supposedly exerted by prefrontal inhibition over premotor regions; see Cross \& Iacoboni, 2014; Cross, Torrisi, Reynolds Losin, \& Iacoboni, 2013). This might be the reason participants did not show in their kinematics any overt sign of simulation of their partner's behavior (i.e., interference). As the exploration of the neural bases of the Sycnhr condition was outside the aims of this study, these issues might be explored in the future.

\section{The Role of aIPS in JA}

In humans, left aIPS plays a crucial role in goal-based grasping planning and control, both during action observation and execution (Tunik et al., 2007). The left aIPS is responsible for goal-based online action control during individual reach-to-grasp movements (Tunik et al., 2005), and it discharges well ahead of a planned hand action, suggesting that this region takes part in the implementation of motor intentions (Desmurget et al., 2009;
Andersen \& Buneo, 2002). Moreover, monkey and human studies have shown that aIPS also codes action goals and intentions in others (Bonini et al., 2010; Jastorff, Begliomini, Fabbri-Destro, Rizzolatti, \& Orban, 2010; Hamilton \& Grafton, 2006; Fogassi et al., 2005). Parietal damage to this region reduces the anticipatory electrophysiological activity arising when healthy individuals expect others to move, indicating that inferior parietal areas are involved in predictive coding of others' actions (Fontana et al., 2012). Finally, the dense anatomical (Schmahmann et al., 2007) and functional (Davare, Kraskov, Rothwell, \& Lemon, 2011; Fogassi \& Luppino, 2005) connections between aIPS and the ventral premotor area found in monkeys and humans make aIPS the ideal candidate for integrating information about the physical environment (including those related with the individual goal) with motor predictions forwarded by the ventral premotor area (e.g., those regarding the partner's goal) during action planning (Tunik et al., 2007).

Thus, aIPS activity during JA might have played a stronger role than during Synchr because only in JA did the observed and executed movements need to be tightly integrated with no help from directly imitating the observed behavior. The JA condition requires fast integration of reliable predictions about the partner's goal within one's own motor plan to rapidly associate the correct response and fulfill the JA goal (in our task, being synchronous and imitative/complementary as a couple). Although this rapid self-other goal integration similarly unfolds in JAs requiring congruent or incongruent actions, the inhibition of aIPS had opposite effects on these two JA conditions in our experiment. We suggest that cTBS over aIPS impaired the integration process, leaving automatic imitation unchanged: This effect results in a dissociation between performance during congruent and incongruent actions because, as previously shown in contexts where the two partners do not share a common goal, they are oppositely affected by automatic imitation (Brass, Bekkering, \& Prinz, 2001; Brass, Bekkering, Wohlschläger, \& Prinz, 2000). In other words, we suggest that the impaired integration associated to aIPS transient inhibition made the JA condition more similar to a nonjoint condition.

Evidence that CTBS over aIPS had no impact on JA motor execution per se, as shown by the absence of significant modulations on RTs or kinematics, suggests that our results were not due to unspecific low-level motor effects. Although some previous studies showed a TMSinduced interference of aIPS stimulation on grasping execution (Rice, Tunik, \& Grafton, 2006; Tunik et al., 2005), these effects were strictly time-locked and occurred only when TMS was delivered over aIPS at specific time windows after perturbation of target orientation. Our offline cTBS protocol, and the absence of online target perturbation, may explain why we did not find a specific effect of aIPS inhibition on individual grasping kinematics. The fact that the present effect of aIPS inhibition was only 
found during the execution of precision grips is also possibly due to the functional properties of aIPS in grasping control. Indeed, this area is more strongly recruited during precision than power grip in humans (Begliomini, Wall, Smith, \& Castiello, 2007; Ehrsson et al., 2000) as a reflection of the need for more online control required by reaching and grasping small objects (Castiello, 2005; Gentilucci et al., 1991, for a review). Furthermore, aIPS inhibition differently affects premotor-motor connectivity in precision and power grips (Davare, Rothwell, \& Lemon, 2010). Previous kinematic studies have also shown that the execution of precision but not power grips is sensitive to high-level cognitive and social manipulations (see Becchio, Manera, Sartori, Cavallo, \& Castiello, 2012, for a review). We suggest that the selective effect found here is likely due to the functional properties of the portion of aIPS we targeted, rather than to intrinsic differences in the cognitive mechanisms at the basis of JAs implying power or precision grips.

\section{Partners' Action Integration and Shared Goals in JA}

From a more theoretical perspective, our results strongly suggest that, during JA, the interdependence between partner's actions modulates motor processes, boosting the role of partner's action integration possibly engaging aIPS activity. As stated in the Introduction, we suggest that the interdependence between partners directly depends on the presence of an overarching JA goal specifying what partners need to do as a couple (e.g., be complementary/imitative) and channeling predictions about what each agent needs to do to achieve this (see also Bekkering et al., 2009). This JA goal is "shared" between partners (Butterfill, 2012, 2016), meaning it is both in common and subdivided between them: Each partner's action (and the related subgoal) is needed to achieve the JA goal. In our task, "being imitative or complementary as a couple" (i.e., the JA goal) can only be the result of the joint activity of two different agents, both performing their own contribution by achieving a specific subgoal (i.e., grasping either the upper or lower target location on the bottle-shaped object; see also Sacheli, Aglioti, et al., 2015). This somehow resembles real-life situations in which, for instance, each partner in a tangoing pair needs to perform his or her own moves to jointly create choreography. Although the virtual partner does not hold any shared goal in our task, participants need to behave under the constraint of his or her movements, which are integral parts of the JA shared goal. Crucially, the presence of this shared goal, which constitutes the only difference between the JA and Synchr conditions, modulates the sensorimotor processes at the basis of interpersonal coordination in our task. Shared goals can thus be defined as sensorimotor representations, taking into account information regarding both the agents' (expected) actions and the environment in which they take place (Sacheli, Aglioti, et al., 2015): This is the reason parietal areas (see also Kokal et al., 2009), and specifically aIPS, play a crucial role.

\section{Conclusions: Motor Hierarchies in JAs}

The theoretical framework described above is slightly in contrast with suggestions that both compatible (congruent) and incompatible (incongruent) stimulus-response associations that occur as a result of visuomotor learning (associative sequence learning account; Catmur, Walsh, \& Heyes, 2009; Heyes, 2001) might account for all human interactive behaviors. Indeed, the key point here is that JAs do not simply imply an agent's response to a partner's action (the stimulus). Similarly, despite that we admit that interpersonal coordination might occur in a variety of social instances that do not necessarily imply any shared goal (Gallotti, Fairhurst, \& Frith, 2017), such as those characterized by involuntary entrainment (Schmidt, Fitzpatrick, Caron, \& Mergeche, 2011), the presence of a shared goal in JAs essentially shapes sensorimotor integration during action planning and singles out JAs from other "social" situations. Indeed, it is the JA shared goal that structures each coagent motor planning: Just as individual muscular synergies are coordinated in complex actions by the need to achieve a desired (individual) motor goal, interpersonal motor synergies are shaped by the presence of shared goals that organize coagents' behaviors (Candidi, Sacheli, \& Aglioti, 2015; Chersi, 2011). Within this line, studies on nonhuman primates showed that most neurons in the parietal cortex (including AIP, the homologous of aIPS) are tuned to higher-order action goal coding (Bonini et al., 2010), which might influence downstream motor planning. We suggest that shared goals might indeed act as higher-level (motor) representations that "actively" bias perceptual processing and modulate predictive motor mechanisms.

\section{Acknowledgments}

The study was supported by a PRIN grant (Progetti di Ricerca di Rilevante Interesse Nazionale, Edit. 2015, Prot. 20159CZFJK) and grant H2020-SESAR-2015-1 (MOTO: The embodied reMOte Tower, project number 699379) to S. M. A. and by a Sapienza University grant (Progetti Medi 2016) to M. C. We would like to thank Dario Sarra for helping in data recording and Enea Francesco Pavone for technical assistance during the experimental setup development.

Reprint requests should be sent to Lucia Maria Sacheli, Department of Psychology and Milan Center for Neuroscience (NeuroMi), University of Milano-Bicocca, Piazza dell'Ateneo Nuovo 1, 20126 Milan, Italy, or via e-mail: lucia.sacheli@unimib. it, or Matteo Candidi, Department of Psychology, "Sapienza" University of Rome, Via dei Marsi 78, 00185, Rome, Italy, or via e-mail: matteo.candidi@uniroma1.it. 


\section{REFERENCES}

Andersen, R. A., \& Buneo, C. A. (2002). Intentional maps in posterior parietal cortex. Anmual Review of Neuroscience. 25, 189-220.

Becchio, C., Manera, V., Sartori, L., Cavallo, A., \& Castiello, U. (2012). Grasping intentions: From thought experiments to empirical evidence. Frontiers in Human Neuroscience, 6, 117.

Begliomini, C., Wall, M. B., Smith, A. T., \& Castiello, U. (2007). Differential cortical activity for precision and whole-hand visually guided grasping in humans. European Journal of Neuroscience, 25, 1245-1252.

Bekkering, H., De Bruijn, E. R., Cuijpers, R. H., NewmanNorlund, R., van Schie, H. T., \& Meulenbroek, R. (2009). Joint action: Neurocognitive mechanisms supporting human interaction. Topics in Cognitive Science, 1, 340-352.

Bonini, L., Rozzi, S., Serventi, F. U., Simone, L., Ferrari, P. F., \& Fogassi, L. (2010). Ventral premotor and inferior parietal cortices make distinct contribution to action organization and intention understanding. Cerebral Cortex, 20, $1372-1385$.

Brass, M., Bekkering, H., \& Prinz, W. (2001). Movement observation affects movement execution in a simple response task. Acta Psychologica, 106, 3-22.

Brass, M., Bekkering, H., Wohlschläger, A., \& Prinz, W. (2000). Compatibility between observed and executed finger movements: Comparing symbolic, spatial, and imitative cues. Brain and Cognition, 44, 124-143.

Briggs, G. G., \& Nebes, R. D. (1975). Patterns of hand preference in a student population. Cortex, 11, 230-238.

Butterfill, S. A. (2012). Joint action and development. Philosophical Ouarterlv, 62, 23-47.

Butterfill, S. A. (2016). Joint action: A minimalist approach. In J. Kiverstein (Ed.), Routledge handbook of philosophy of the social mind (pp. 357-369). London: Routledge.

Candidi, M., Curioni, A., Donnarumma, F., Sacheli, L. M., \& Pezzulo, G. (2015). Interactional leader-follower sensorimotor communication strategies during repetitive joint actions. Journal of the Roval Societv Interface, 12, 20150644

Candidi, M., Sacheli, L. M., \& Aglioti, S. M. (2015). From muscles synergies and individual goals to interpersonal synergies and shared goals: Mirror neurons and interpersonal action hierarchies: Comment on "Grasping synergies: A motorcontrol approach to the mirror neuron mechanism" by D'Ausilio et al. Physics of Life Reviews, 12, 126-128.

Castiello, U. (2005). The neuroscience of grasping. Nature Reviews Neuroscience, 6, 726-736.

Catmur, C., Walsh, V., \& Heyes, C. (2009). Associative sequence learning: The role of experience in the development of imitation and the mirror system. Philosophical Transactions of the Royal Society of London, Series B: Biological Sciences, 364, 2369-2380.

Chersi, F. (2011). Neural mechanisms and models underlying joint action. Experimental Brain Research, 211, 643-653.

Corbetta, M., \& Shulman, G. L. (2002). Control of goal-directed and stimulus-driven attention in the brain. Nature Reviews Neuroscience, 3, 201-215.

Cross, K. A., \& Iacoboni, M. (2014). Neural systems for preparatory control of imitation. Philosophical Transactions of the Royal Society of London, Series B: Biological Sciences, 369, 20130176.

Cross, K. A., Torrisi, S., Reynolds Losin, E. A., \& Iacoboni, M. (2013). Controlling automatic imitative tendencies:

Interactions between mirror neuron and cognitive control systems. Neuroimage, 83, 493-504.

D’Ausilio, A., Badino, L., Li, Y., Tokay, S., Craighero, L., Canto, R., et al. (2012). Leadership in orchestra emerges from the causal relationships of movement kinematics. PLoS One, 7, e35757.

Davare, M., Kraskov, A., Rothwell, J. C., \& Lemon, R. N. (2011). Interactions between areas of the cortical grasping network. Current Opinion in Neurobiology, 21, 565-570.

Davare, M., Rothwell, J. C., \& Lemon, R. N. (2010). Causal connectivity between the human anterior intraparietal area and premotor cortex during grasp. Current Biology, 20, 176-181.

Desmurget, M., Reilly, K. T., Richard, N., Szathmari, A., Mottolese, C., \& Sirigu, A. (2009). Movement intention after parietal cortex stimulation in humans. Science, 324, 811-813.

Ehrsson, H. H., Fagergren, A., Jonsson, T., Westling, G., Johansson, R. S., \& Forssberg, H. (2000). Cortical activity in precision- versus power-grip tasks: An fMRI study. Lournal of Neuropbysiology, 83, 528-536.

Fogassi, L., Ferrari, P. F., Gesierich, B., Rozzi, S., Chersi, F., \& Rizzolatti, G. (2005). Parietal lobe: From action organization to intention understanding. Science, 308, 662-667.

Fogassi, L., \& Luppino, G. (2005). Motor functions of the parietal lobe. Current Opinion in Neurobiology, 15, 626-631.

Fontana, A. P., Kilner, J. M., Rodrigues, E. C., Joffily, M., Nighoghossian, N., Vargas, C. D., et al. (2012). Role of the parietal cortex in predicting incoming actions. Neuroimage. 59, 556-564.

Gallotti, M., Fairhurst, M. T., \& Frith, C. D. (2017). Alignment in social interactions. Consciousness and Cognition, 48, 253-261.

Gentilucci, M., Castiello, U., Corradini, M. L., Scarpa, M., Umiltà, C., \& Rizzolatti, G. (1991). Influence of different types of grasping on the transport component of prehension movements. Neuropsychologia, 29, 361-378.

Grafton, S. T. (2010). The cognitive neuroscience of prehension: Recent developments. Experimental Brain Research, 204, 475-491.

Hamilton, A. F., \& Grafton, S. T. (2006). Goal representation in human anterior intraparietal sulcus. Journal of Neuroscience, 26, 1133-1137.

Heyes, C. (2001). Causes and consequences of imitation. Trends in Cognitive Sciences, 5, 253-261.

Heyes, C. (2011). Automatic imitation. Psychological Bulletin. 137, 463-483

Huang, Y. Z., Edwards, M. J., Rounis, E., Bhatia, K. P., \& Rothwell, J. C. (2005). Theta burst stimulation of the human motor cortex. Neuron, 45, 201-206.

Iacoboni, M. (2005). Neural mechanisms of imitation. Current Opinion in Neurobiologv, 15, 632-637.

Jastorff, J., Begliomini, C., Fabbri-Destro, M., Rizzolatti, G., \& Orban, G. A. (2010). Coding observed motor acts: Different organizational principles in the parietal and premotor cortex of humans. Journal of Neuropbysiologv, 104, 128-140.

Keysers, C., \& Gazzola, V. (2014). Hebbian learning and predictive mirror neurons for actions, sensations and emotions. Philosophical Transactions of the Royal Society of London, Series B: Biological Sciences, 369, 20130175.

Knoblich, G., \& Jordan, J. S. (2003). Action coordination in groups and individuals: Learning anticipatory control. Journal of Experimental Psychology: Learning. Memorv. and Cognition, 29, 1006-1016.

Kokal, I., Gazzola, V., \& Keysers, C. (2009). Acting together in and beyond the mirror neuron system. Neuroimage, 47 , 2046-2056.

Love, J., Selker, R., Marsman, M., Jamil, T., Verhagen, A. J., \& Ly, A. (2015). JASP (Version 0.6.6) [Computer software]. https://jasp-stats.org

Newman-Norlund, R. D., van Schie, H. T., van Zuijlen, A. M. J., \& Bekkering, H. (2007). The mirror neuron system is more active during complementary compared with imitative action. Nature Neuroscience, 10, 817-818. 
Ocampo, B., \& Kritikos, A. (2010). Placing actions in context: Motor facilitation following observation of identical and non-identical manual acts. Experimental Brain Research. 201, 743-751.

Rice, N. J., Tunik, E., \& Grafton, S. T. (2006). The anterior intraparietal sulcus mediates grasp execution, independent of requirement to update: New insights from transcranial magnetic stimulation. Iournal of Neuroscience, 26, 8176-8182.

Rossi, S., Hallett, M., Rossini, P. M., Pascual-Leone, A., \& The Safety of TMS Consensus Group. (2009). Safety, ethical considerations, and application guidelines for the use of transcranial magnetic stimulation in clinical practice and research. Clinical Neurophysiologv, 120, 2008-2039.

Rouder, J. N., Morey, R. D., Verhagen, J., Swagman, A. R., \& Wagenmakers, E.-J. (2017). Bayesian analysis of factorial designs. Psvchological Methods, 22, 304-321.

Sacheli, L. M., Aglioti, S. M., \& Candidi, M. (2015). Social cues to joint actions: The role of shared goals. Frontiers in Psvchology, 6, 1034.

Sacheli, L. M., Candidi, M., Era, V., \& Aglioti, S. M. (2015). Causative role of left aIPS in coding shared goals during human-avatar complementary joint actions. Nature Communications, 6, 7544.

Sacheli, L. M., Candidi, M., Pavone, E. F., Tidoni, E., \& Aglioti, S. M. (2012). And yet they act together: Interpersonal perception modulates visuo-motor interference and mutual adjustments during a joint-grasping task. PLoS One, 7, e50223.

Sacheli, L. M., Christensen, A., Giese, M. A., Taubert, N., Pavone, E. F., Aglioti, S. M., et al. (2015). Prejudiced interactions: Implicit racial bias reduces predictive simulation during joint action with an out-group avatar. Scientific Reports. 5, 8507.

Sacheli, L. M., Tidoni, E., Pavone, E. F., Aglioti, S. M., \& Candidi, M. (2013). Kinematics fingerprints of leader and follower role-taking during cooperative joint actions. Experimental Brain Research, 226, 473-486.
Schmahmann, J. D., Pandya, D. N., Wang, R., Dai, G., D'Arceuil, H. E., de Crespigny, A. J., et al. (2007). Association fibre pathways of the brain: Parallel observations from diffusion spectrum imaging and autoradiography. Brain, 130, 630-653.

Schmidt, R. C., Fitzpatrick, P., Caron, R., \& Mergeche, J. (2011). Understanding social motor coordination. Human Movement Science, 30, 834-845.

Sebanz, N., Bekkering, H., \& Knoblich, G. (2006). Joint action: Bodies and minds moving together. Trends in Cognitive Sciences, 10, 70-76.

Talairach, J., \& Tournoux, P. (1988). Co-planar stereotaxic atlas of the buman brain. 3-dimensional proportional system: An approach to cerebral imaging. New York: Thieme Medical Publishers.

Tieri, G., Tidoni, E., Pavone, E. F., \& Aglioti, S. M. (2015). Mere observation of body discontinuity affects perceived ownership and vicarious agency over a virtual hand. Experimental Brain Research, 233, 1247-1259.

Tunik, E., Frey, S. H., \& Grafton, S. T. (2005). Virtual lesions of the anterior intraparietal area disrupt goal-dependent on-line adjustments of grasp. Nature Neuroscience, 8, 505-511.

Tunik, E., Rice, N. J., Hamilton, A., \& Grafton, S. T. (2007). Beyond grasping: Representation of action in human anterior intraparietal sulcus. Neuroimage, 36, T77-T86.

van Schie, H. T., van Waterschoot, B. M., \& Bekkering, H. (2008). Understanding action beyond imitation: Reversed compatibility effects of action observation in imitation and joint action. Journal of Experimental Psychologv: Human Perception and Performance, 34, 1493-1500.

Vesper, C., van der Wel, R. P. R. D., Knoblich, G., \& Sebanz, N. (2013). Are you ready to jump? Predictive mechanisms in interpersonal coordination. Journal of Experimental Psucholog: Human Percebtion and Performance, 39, 48-61. Wang, L., Liu, X., Guise, K. G., Knight, R. T., Ghajar, J., \& Fan, J. (2010). Effective connectivity of the fronto-parietal network during attentional control. Journal of Cognitive Neuroscience, 22, 543-553. 